SLEEP DISORDERED BREATHING

\title{
Randomised controlled crossover trial of humidified continuous positive airway pressure in mild obstructive sleep apnoea
}

\author{
N S Marshall, A M Neill, A J Campbell, D S Sheppard
}

Thorax 2005;60:427-432. doi: 10.1136/thx.2004.032078

See end of article for authors' affiliations

Correspondence to:

Dr A Neill, Wellsleep, Department of Medicine, Wellington School of Medicine and Health Sciences, University of Otago, Wellington, New Zealand; aneill@wnmeds. ac.nz

Received 25 July 2004 Accepted 7 March 2005

\begin{abstract}
Background: It is unclear whether continuous positive airway pressure (CPAP), the treatment of choice for severe obstructive sleep apnoea (OSA), is effective at improving outcomes in mild OSA.

Methods: To help define the role of humidified CPAP in mild OSA, a randomised crossover study was undertaken of patients with an apnoea hypopnoea index (AHI) of 5-30/hour. Subjective sleepiness, objective wakefulness, mood, reaction time, and quality of life were measured at baseline, after 3 weeks treatment with humidified CPAP and 3 weeks sham CPAP (2 week washout).

Results: Twenty nine of 31 enrolled patients (age 25-67 years, seven women, mean (SD) body mass index $31.5(6) \mathrm{kg} / \mathrm{m}^{2}$ ) completed the protocol. Humidified CPAP improved polysomnographic indices of OSA and Epworth Sleepiness Scale (2.4 points (95\% Cl 0.6 to 4.2)). Objective wakefulness (modified maintenance of wakefulness test) showed a trend towards improvement $(5.2$ minutes $(95 \% \mathrm{Cl}-0.6$ to 11)). Mood (Hospital Anxiety and Depression Scale), quality of life (SF 36, Functional Outcomes of Sleep Questionnaire), and reaction times (Psychomotor Vigilance Task) were not improved more than sham CPAP. Compliance with humidified and sham CPAP both averaged 4.9 hours/night. Placebo effects were evident in many outcomes and there was no clear treatment preference.

Conclusions: Humidified CPAP improves subjective sleepiness and possibly objective wakefulness but not reaction times, quality of life, or mood. These results do not support the routine use of CPAP in all patients with mild OSA, but offers some support for the trialling of CPAP in those with severe sleepiness.
\end{abstract}

O bstructive sleep apnoea syndrome (OSA) is a common sleep breathing disorder affecting $2-4 \%$ of the middle aged population. ${ }^{1}$ Most people with OSA have disease of mild to moderate severity with polysomnographically defined apnoea hypopnoea indices of between 5 and 30/hour (hereafter called mild). ${ }^{1}$ Continuous positive airway pressure (CPAP) is an effective treatment for severe presentations of the syndrome. ${ }^{2}{ }^{3}$ However, despite six randomised controlled trials, the role of CPAP in the treatment of mild OSA remains clinically uncertain..$^{4-9}$ Potential limitations of these studies include suboptimal compliance averaging 2.8 to 4.8 hours/ night,,$^{78}$ moderate dropout rates of $8 \%$ to $33 \%,{ }^{5}$ and the use of different treatment comparisons ${ }^{10}{ }^{11}$ including orally ingested placebo pill $^{4-7}$ and conservative management advice. ${ }^{8}{ }^{9}$ Humidification of CPAP has been shown to improve adverse upper airway symptoms and initial compliance. ${ }^{12}{ }^{13} \mathrm{~A}$ crossover trial structure is arguably superior for investigating a chronic condition such as OSA but has not previously been combined with sham CPAP. By using a sham CPAP device to mimic CPAP and following identical treatment procedures, it is possible to successfully maintain blinding, ${ }^{14}$ even in a crossover trial.

The current study aimed to assess the effectiveness of humidified nasal CPAP on mild OSA using a blinded, randomised, sham CPAP controlled crossover trial measuring clinically relevant changes in daytime sleepiness, mood, quality of life, and psychomotor function while measuring the effects of compliance.

\section{METHODS}

Study design and inclusion criteria

The protocol was approved by the Wellington ethics committee. Each arm of the trial was 3 weeks long with a 2 week washout period between arms included to control for carryover effects. Data were collected between November 2001 and February 2003. The number of patients in each treatment group was derived from simple coin flipping. Treatment allocation sequence was not predetermined and was achieved by the duty polysomnographic technician blindly drawing a slip of paper without replacement from an urn after testing on the first day had been completed. Patients were randomised to receive either sham CPAP or fully titrated humidified CPAP (Fisher and Paykel HC 221; Fisher and Paykel Healthcare, Auckland, New Zealand) for 3 weeks. Patients were informed that the study was "testing two different pressures of humidified CPAP". The investigator responsible for daytime study data collection (NSM) was blinded to treatment allocation.

English speaking patients, naïve to CPAP, referred from local sleep clinics were prospectively screened for study inclusion if diagnostic polysomnography showed an apnoea hypopnoea index (AHI) of 5-30/hour. Patients were accepted if they were older than 18 years and complained of habitual snoring or nocturnal choking and at least one daytime sleepiness symptom (daytime/evening napping, sleepiness while driving, never or rarely awakening refreshed) or had an Epworth Sleepiness Score $\geqslant 8$ and gave written informed consent to be in the trial. Clinical examination, full blood cell count, electrolytes, renal function, random blood glucose and liver function tests were undertaken to exclude medical co-morbidities.

Diagnostic polysomnography was undertaken either in a tertiary level sleep laboratory (WellSleep, Bowen Hospital) or in the patients' own home (S Series or P Series Sleep System, Compumedics, Melbourne, Australia) in accordance with accepted scientific methodology. ${ }^{15}$ At least 4 hours of sleep, including at least 30 minutes of REM sleep and 30 minutes sleeping in the supine position were required. Patients were 
excluded if they had a history of extreme somnolence requiring immediate treatment; performed shift work; had chronic sleep restriction (average total sleep time $\leqslant 6$ hours/ night); were currently taking sedatives, antidepressants, psychotropics or stimulants; had an alcohol intake of $>3$ standard units/24 hours or caffeine dependency (unable to forego caffeine on testing days); had undergone upper airway surgery since the diagnostic sleep study; or had any clinically significant co-existing disease or additional sleep disorders.

It was calculated that 31 patients would be required to show a 2.5 point improvement between sham CPAP and CPAP in the Epworth Sleepiness Scale with 90\% power, significance set at 0.05 and an intra-individual SD of 4.1 points in a crossover design. ${ }^{6}$

\section{Daytime procedure}

Testing began at 12.30 hours on each of the four study days at the beginning and end of each treatment arm. All testing procedures were time of day fixed. Overnight polysomnography was undertaken at the beginning of each arm for either CPAP titration or to determine the effect of the sham CPAP device.

\section{Polysomnography and analysis}

Comprehensive polysomnography included an electroencephalogram $\left(\mathrm{C}_{4}-\mathrm{A}_{1} / \mathrm{C}_{3}-\mathrm{A}_{2}\right)$, left and right electro-oculogram, submental electromyogram, abdominal and thoracic respiratory effort (piezoelectric bands), left/right leg movements, body position, pulse oximetry, oronasal airflow (by pressure transducer and thermistor), electrocardiogram and, where appropriate, CPAP mask pressure. Studies were scored in 30 second epochs by an experienced sleep scientist (AJC, DSS) using accepted international criteria. ${ }^{16}{ }^{17}$

The AHI was calculated by summing the total number of apnoeas and hypopnoeas and dividing by the number of hours of sleep. An obstructive apnoea was defined as cessation of breathing for 10 seconds or more $(<20 \%$ normal airflow) with ongoing respiratory effort. Without this effort a 10 second apnoea or longer was defined as a central apnoea; combinations of effort/no effort defined a mixed apnoea. Hypopnoea was defined as greater than $50 \%$ reduction in the amplitude of at least two of the three respiratory channels (oronasal flow, thoracic, and abdominal effort) or $>50 \%$ reduction in nasal pressure for at least 10 seconds accompanied by either an arousal from sleep or $\geqslant 4 \%$ blood oxygen dip.

\section{Continuous positive airway pressure systems}

Therapeutic CPAP was determined by manual titration to abolish apnoeas, hypopnoeas, and reduce respiratory related arousals and confirmed by a sleep physician (AMN). The sham $\mathrm{CPAP}^{14}$ device was set to $8 \mathrm{~cm} \mathrm{H}_{2} \mathrm{O}$ but actually delivered $<1.0 \mathrm{~cm} \mathrm{H}_{2} \mathrm{O}$ due to placement of a resistor at the pump outlet and a modified mask with extra holes drilled around the exhalation port (Mirage, ResMed, Sydney, Australia). The sham CPAP created conditions that were identical to CPAP at $8 \mathrm{~cm} \mathrm{H}_{2} \mathrm{O}$ in terms of noise, mask temperature, mask humidity, and airflow through the exhalation port.

\section{Outcome measures}

\section{Sleepiness}

The Epworth Sleepiness Scale $(\mathrm{ESS})^{18}$ and modified Maintenance of Wakefulness Test (modMWT) ${ }^{19}{ }^{20}$ were used. To enhance patient recruitment and study completion we abbreviated the Maintenance of Wakefulness Test to include two 40 minute nap opportunities which were started at 13.30 and 15.30 hours. Three consecutive 30 second epochs of stage
1 or one epoch of stage 2 or higher indicated sleep onset and was measured by live visual scoring of the EEG.

\section{Quality of life}

The Functional Outcomes of Sleepiness Questionnaire $(\mathrm{FOSQ})^{21}$ and Short Form of the Medical Outcomes Survey $(\mathrm{SF}-36)^{22}{ }^{23}$ were used. The FOSQ scale was employed without the sexual functioning subscale in order to avoid differences in comparison between patients who were and were not in sexual relationships.

Mood

Mood was measured using the Hospital Anxiety and Depression Scale (HADS). ${ }^{24}$

\section{Psychomotor performance}

The Psychomotor Vigilance Task (PVT) ${ }^{25}$ was administered twice a day with a 1 minute practice session before each 10 minute data collection session at 14.30 and 16.30 hours.

\section{Compliance with treatment}

Compliance with treatment was objectively measured by an internal pressure sensor and expressed as the average number of hours of use per night (HC 221, Compliance Maximiser release 1.01; Fisher and Paykel Healthcare, Auckland New Zealand). The active CPAP compliance was the time on the face at pressure, and sham CPAP compliance was the machine run time.

\section{Treatment preference}

At the end of the trial, patients were asked which treatment was preferred and which was best for their sleep.

\section{Data handling and statistical analyses}

Significance and size of treatment effects were tested in SAS (SAS Institute, version 8) after double data entry. Paired sample $t$ tests were used to detect changes from baseline sleep variables (or normal transformations) due to sham CPAP and to test for carryover effects between the start of each treatment arm. $\chi^{2}$ tests were used to test for differences in patient preferences between CPAP and placebo. Mixed model analyses of variance were employed to investigate treatment induced improvements. Results for the modMWT and PVT were averaged within the day. CPAP compliance was split at 4 hours per night and used to classify patients as high or low compliers. Treatment, order of treatment, compliance and interactions between treatment by compliance were fixed effects; individuals were random effects. Main effects and any interactions were regarded as statistically significant when $\mathrm{p}<0.05$. Main effects were interpreted as favouring treatment when CPAP was significantly better than sham CPAP and the pattern from the treatment by compliance interaction subanalyses indicated a significant benefit of high compliance on CPAP over both sham CPAP compliance combinations. Effect sizes were used to verify the magnitude of the net treatment induced changes. They were calculated by dividing each mean net effect by the standard deviation of the baseline distribution for that measure. Small effect sizes were between 0.20 and 0.50 , medium 0.50 and 0.80 , and large effect sizes $>0.80 .{ }^{26}$ All analyses were undertaken under the intention to treat principle.

\section{RESULTS}

\section{Patient characteristics and retention}

Thirty one of the 53 eligible patients (58.5\%) agreed to participate. Non-participants were not significantly different in terms of age, sex, body mass indices, referral source, or AHI. Two of the 31 patients dropped out after randomisation, both in the first arm (fig 1). One suffered a non-fatal 


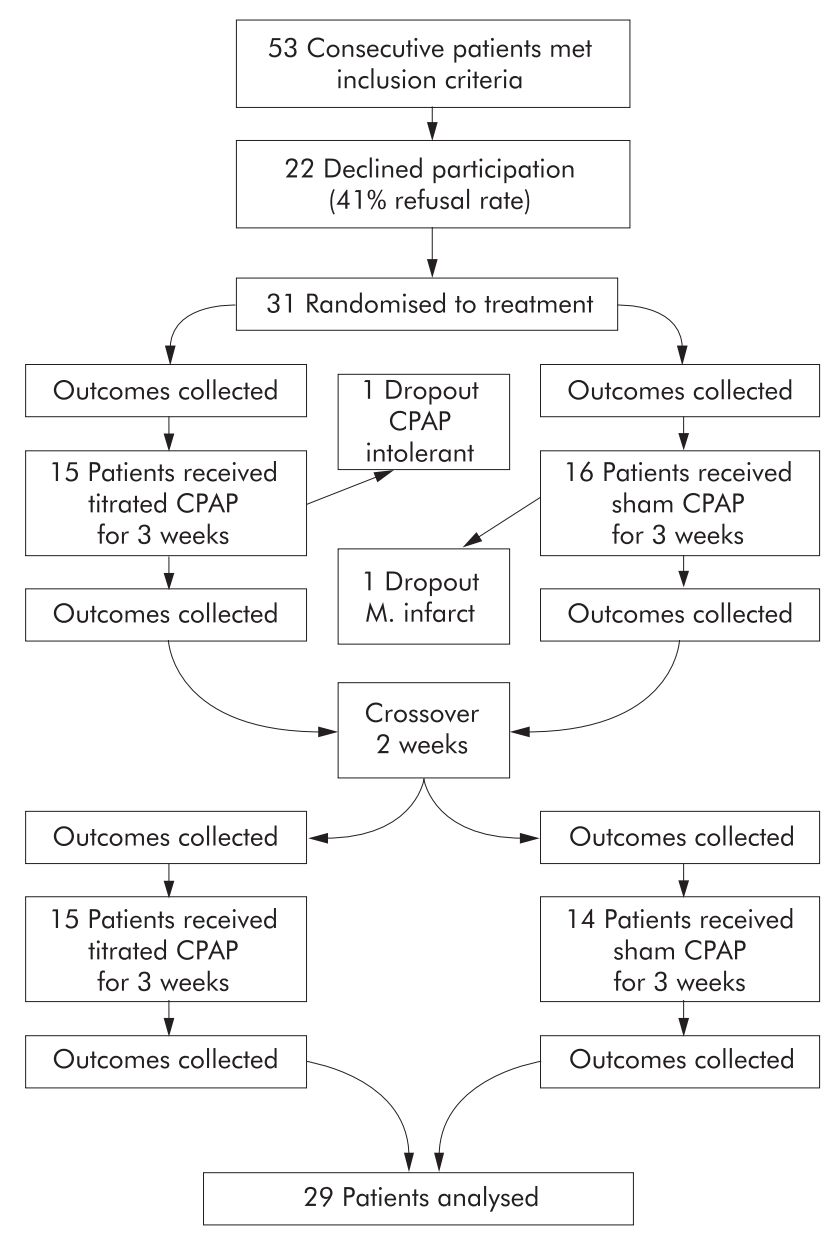

Figure 1 Trial flow chart.

myocardial infarction during sham treatment and the other was CPAP intolerant. Patients were on average overweight (mean (SD) body mass index $31.5(6.0) \mathrm{kg} / \mathrm{m}^{2}$ ), middle aged (mean 50.5 years, range 25-67), and predominantly male $(\mathrm{n}=22,76 \%)$.

\section{Polysomnography at baseline and effects of placebo CPAP}

Baseline polysomnography findings are shown in table 1 together with the full night effects of sham CPAP. Compared

Table 1 Effects of a sham CPAP device on polysomnographic variables

\begin{tabular}{lll}
\hline Sleep variable & Diagnostic night & Placebo night \\
\hline Sleep efficiency (\%) & $84.5(8.9)$ & $79.2(11.7)^{\star}$ \\
Sleep length (min) & $364(74)$ & $357(69)$ \\
Sleep latency (min) & $10.1(11.3)$ & $21.3(16.6)^{\star *}$ \\
Arousal index (/hour) & $25.1(7.1)$ & $22.2(9.8)$ \\
$\mathrm{SaO}_{2}$ lowest point (\%) & $85.7(3.7)$ & $85.1(6.5)$ \\
$\mathrm{Mean} \mathrm{SaO}_{2}$ desat (\%) & $3.6(1.1)$ & $3.7(1.7)$ \\
Total AHI (/hour) & $21.6(7.5)$ & $22.0(14.5)$ \\
Supine sleep (min) & $127(88)$ & $175(99)^{*}$ \\
Stage 1 sleep (\%) & $10.8(5.3)$ & $9.0(4.7)$ \\
Stage 2 sleep (\%) & $46.3(8.3)$ & $56.5(7.7)^{\star *}$ \\
Stage 3/4 sleep (\%) & $23.3(10.7)$ & $17.5(6.5)^{\star *}$ \\
Stage REM (\%) & $19.6(6.3)$ & $17.0(5.6)$ \\
\hline
\end{tabular}

$\mathrm{AHI}$, apnoea-hypopnoea index; $\mathrm{SaO}_{2}$, oxygen saturation. Values are mean (SD).

${ }^{*} p<0.05,{ }^{* *} p<0.01$ : significant differences within patients compared with the diagnostic night.

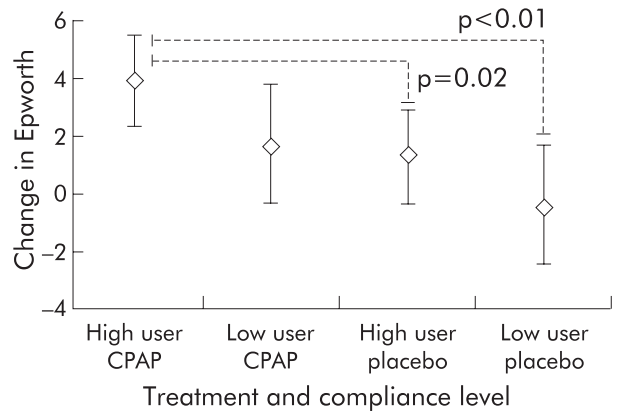

Figure 2 Effects of treatment and compliance on the Epworth Sleepiness Scale. Diamonds indicate mean effects; extended vertical bars are the $95 \%$ confidence limits of the means. The figure shows that subjects with a high rate of compliance with CPAP use had improvements in sleepiness that were better than those with a high or low rate of compliance with sham CPAP.

with baseline, sham treatment increased sleep latency, the percentage of stage 2 sleep, and mildly reduced sleep efficiency. There were no significant effects on the total AHI or arousal index.

\section{Main effects of treatment}

The main effects of CPAP and sham CPAP on all outcome measures are shown in table 2. No carryover effects were observed. Humidified CPAP improved subjective sleepiness (measure by the Epworth Sleepiness Scale). This effect size of humidified CPAP on Epworth scores (SD 4.1) was moderate at 0.58 ( 2.39 points, $95 \%$ CI 0.6 to 4.2 ). There was evidence of a dose-response relationship with subjects with a high compliance rate ( $\geqslant 4$ hours/night) improving by a mean (SE) of $2.6(0.6)$ points while those with a low rate of compliance improved by only $0.7(0.7)$ points $(p=0.04)$. The effects of treatment and compliance were additive; high compliance on CPAP (3.9 points) was better than both low compliance (0.4) and high compliance (1.3) on sham CPAP (fig 2).

Objective wakefulness (modified MWT) exhibited a trend towards improvement for CPAP over sham CPAP (5.2 minutes (95\% CI -0.6 to 11$)$ ) with a small effect size $(0.40)$. Mood (Hospital Anxiety and Depression Scale), quality of life (SF 36, Functional Outcomes of Sleep Questionnaire), and reaction times (Psychomotor Vigilance Task) were not improved by CPAP. Sham effects were observed in many of the measures of quality of life and mood (table 2). In the PVT mean reaction time an interaction was observed between compliance and treatment $(\mathrm{p}=0.03)$ due to deterioration by poor compliers on CPAP treatment $(-14$ (8) ms, $\mathrm{p}=0.07)$ while other groupings improved non-significantly. A significant order effect indicated a slight improvement on the first arm $(10 \mathrm{~ms})$ and slight worsening on the second arm ( $-7 \mathrm{~ms})$.

Titrated CPAP pressure ranged between 5 and $10 \mathrm{~cm} \mathrm{H}_{2} \mathrm{O}$. Average compliance with humidified CPAP was objectively measured at 4.9 hours/night (range 0-8.4 hours). Eighteen patients $(62 \%)$ used the device more than 4 hours/night, and $16(55 \%)$ used the device on average more than 6 hours/ night. Average compliance on sham CPAP was also 4.9 hours/ night (range 0-8.32 hours) and followed a very similar pattern to compliance with humidified CPAP.

General patient preference favoured neither treatment, with 17 of 29 preferring placebo $\left(\chi^{2}=0.86, p=0.35\right)$. Nineteen thought that sham treatment was the most beneficial for their sleep $\left(\chi^{2}=2.79, p=0.09\right)$. Baseline Epworth scores were positively correlated with the sham 
Table 2 Summary of mixed models comparing the effects of humidified CPAP with sham CPAP and effects of order, compliance, and interactions

\begin{tabular}{|c|c|c|c|c|c|c|}
\hline $\begin{array}{l}\text { Outcome measurement (and } \\
\text { maximum score or standard } \\
\text { unit size) }\end{array}$ & $\begin{array}{l}\text { Baseline } \\
\text { Mean (SE) }\end{array}$ & $\begin{array}{l}\text { Placebo } \\
\text { Mean (SE) }\end{array}$ & $\begin{array}{l}\text { CPAP } \\
\text { Mean (SE) }\end{array}$ & $\begin{array}{l}\text { Net effect } \\
(95 \% \mathrm{Cl})\end{array}$ & Effect size & $\begin{array}{l}\text { CPAP usage } \\
\text { (p value) }\end{array}$ \\
\hline Epworth Scale (24) & $12.5(0.8)$ & $12.0(0.7)$ & $9.7(0.7)^{* * *}$ & $2.4(0.6$ to 4.2$)$ & 0.58 & 0.04 \\
\hline ModMWT (latency, min) & $20.9(2.5)$ & $17.9(2.0)$ & $23.1(2.0)$ & $5.2(-0.6$ to 11$)$ & 0.40 & 0.09 \\
\hline FOSQ Total (16) & $12.6(0.3)$ & $13.3(0.3)$ & $13.6(0.3)^{* *}$ & $0.3(-0.5$ to 1.1$)$ & 0.14 & 0.96 \\
\hline Activity (4) & $3.0(0.1)$ & $3.3(0.1)^{*}$ & $3.3(0.1)^{* *}$ & $0.1(-0.1$ to 0.3$)$ & 0.02 & 0.82 \\
\hline Social outcomes (4) & $3.2(0.1)$ & $3.5(0.1)^{*}$ & $3.7(0.1)^{\star \star}$ & $0.2(-0.2$ to 0.5$)$ & 0.17 & 0.20 \\
\hline Vigilance (4) & $3.0(0.1)$ & $3.1(0.1)$ & $3.2(0.1)$ & $0.1(-0.2$ to 0.3$)$ & 0.13 & 0.05 \\
\hline General product (4) & $3.3(0.1)$ & $3.4(0.1)$ & $3.4(0.1)$ & $0.0(-0.2$ to 0.2$)$ & 0.13 & 0.65 \\
\hline \multicolumn{7}{|l|}{ SF-36 (all/100) } \\
\hline Mental health & $75(3)$ & $80(2)^{*}$ & $77(2)$ & $-3(-10$ to 3$)$ & -0.22 & 0.74 \\
\hline Bodily pain & $73(4)$ & $75(4)$ & $68(4)$ & $-7(-17$ to 3$)$ & -0.31 & 0.04 \\
\hline Social functioning & 79 (4) & $85(5)$ & 78 (5) & $-8(-21$ to 6$)$ & -0.36 & 0.26 \\
\hline Vitality & 44 (3) & $53(3)^{* *}$ & $53(3)^{* *}$ & $-1(-10$ to 9$)$ & -0.04 & 0.12 \\
\hline Role emotional & $78(7)$ & 89 (7) & 77 (7) & $-12(-32$ to 9$)$ & -0.30 & 0.82 \\
\hline Role physical & $63(8)$ & $82(7)^{* *}$ & $66(7)$ & $-16(-35$ to 3$)$ & -0.38 & 0.85 \\
\hline Physical functioning & $82(3)$ & $80(2)$ & $81(2)$ & $1(-3$ to 6$)$ & 0.09 & 0.02 \\
\hline General health & $74(3)$ & $76(2)$ & $76(2)$ & $0(-6$ to 7$)$ & 0.03 & 0.46 \\
\hline HADS-Anxiety (21) & $6.8(0.7)$ & $5.7(0.4)^{* *}$ & $6.5(0.4)$ & $-0.8(-1.9$ to 0.4$)$ & -0.20 & 0.52 \\
\hline HADS-Depression (21) & $4.2(0.5)$ & $3.5(0.3)^{\star}$ & $3.8(0.3)$ & $-0.3(-1.2$ to 0.6$)$ & -0.10 & 0.29 \\
\hline \multicolumn{7}{|l|}{ Psychomotor vigilance task } \\
\hline Mean RT (ms) & $264(5)$ & $259(5)$ & $266(5)$ & $-7(-20$ to 7$)$ & -0.15 & 0.16 \\
\hline Lapses (>500 ms RT) & $1.3(0.3)$ & $1.0(0.4)$ & $1.3(0.4)$ & $-0.4(-1.4$ to 0.7$)$ & -0.18 & 0.15 \\
\hline Errors & $2.8(0.5)$ & $3.3(0.7)$ & $3.2(0.7)$ & $0.1(-1.9$ to 2.0$)$ & 0.02 & 0.27 \\
\hline
\end{tabular}

ModMWT, Modified Maintenance of Wakefulness test; FOSQ, Functional Outcomes of Sleepiness Scale; SF-36, Short Form 36 Question version of the Medical Outcomes Survey; HADS, Hospital Anxiety and Depression Scale; RT, reaction time.

Measures (maximum score) with mean (SE) values at baseline and for the effects of CPAP and placebo. ${ }^{*} p<0.05$; ${ }^{* *} p<0.01$; ${ }^{* * *} p<0.001$ compared with baseline. A positive net effect indicates that CPAP outperformed sham CPAP and negative effect sizes indicate that placebo outperformed CPAP. CPAP usage column indicates simple dose-response relationships. Only the Vitality subscale of the SF-36 and the PVT mean RT exhibited any order effect. Mean (SE) values are derived from within the mixed models. Baseline values are from the first day of testing.

CPAP adjusted improvements in Epworth after CPAP treatment (Pearson's $r=0.48, \mathrm{p}<0.01$; fig 3).

\section{DISCUSSION}

In patients with mild OSA, humidified CPAP reduced subjective sleepiness compared with sham CPAP. Objective wakefulness was also improved, but this effect did not reach significance and was likely to be subject to a type II error. CPAP did not improve reaction time, mood, or quality of life. Statistically significant placebo effects were observed in a number of the subjective outcome measures. Significant strengths of the study include the use of humidification as an adjuvant to CPAP, a low level of drop outs $(6.5 \%)$, relatively high compliance ( 4.9 hours/night), and the novel use of sham CPAP in a crossover trial.

Potential explanations for the lack of significant improvements due to CPAP therapy in the majority of outcome measures include: a small numbers of participants (type II error), lack of initial impairment, suboptimal compliance,

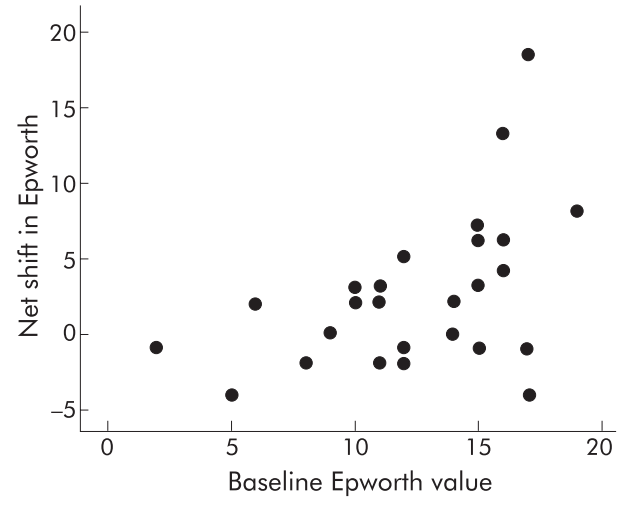

Figure 3 Baseline Epworth values predict response to treatment after controlling for placebo and regression to the mean effects. insufficient treatment duration, or that CPAP is not an effective treatment for patients with mild OSA.

Type II errors were a potential problem with this study. Using the observed differences and variability with $80 \%$ power and a significance level of $5 \%$ in a crossover design, only two of the 19 non-significant outcome measures indicated that a reasonable number of extra patients would have been required to show the observed difference. The modMWT would have required 38 patients and the FOSQ social outcomes scale 64 patients to show the observed differences were significant. However, most of the observed modMWT effect was due to a worsening on the sham arm and would not be observed in clinical practice (table 2). All other outcomes either favoured sham CPAP over CPAP $(n=10)$ or would have required more than 340 patients (three required many thousands) to show significance. The vast numbers of people required to show these tiny differences lends strength to the conclusion that the effects observed are largely indistinguishable from zero and are not evidence of type II errors.

Twenty mixed models were run to test for the efficacy of CPAP therapy; improvements in subjective sleepiness might have been a type I error but, as the improvement fitted within an hypothesised dose-response relationship and is consistent with the known effects of CPAP in severe OSA, ${ }^{3}$ the result is likely to be clinically relevant. The effect size for the Epworth Sleepiness Scale (0.58) and the Maintenance of Wakefulness Test $(0.40)$ were moderate to small, indicating potential clinical significance.

Our patient group exhibited sleep related morbidity, potentially amenable to treatment, with significant decrements compared with the normative population estimates of the Epworth Sleepiness Scale, ${ }^{27}$ Maintenance of Wakefulness Test, ${ }^{19}{ }^{20}$ and in some subscales of the Functional Outcomes of Sleepiness ${ }^{21}$ and the SF- $36 .^{23}$

Treatment durations in previous studies of mild OSA have ranged from 4 weeks to 6 months, while this study used 3 week arms. The two trials ${ }^{67}$ employing 4 week treatment 
durations showed both treatment and placebo effects, while trials of only 1 week CPAP in a broad spectrum of OSA have also shown benefits to $\operatorname{mood}^{28}$ and neuropsychological function..$^{29}$ The onset of CPAP induced reduction in sleepiness occurs within a single night of treatment and is further improved at 2 weeks but not after 6 weeks. ${ }^{30}$ In this study 3 weeks of treatment resulted in improvements in sleepiness in addition to a number of sham CPAP effects in mood and quality of life measures, indicating sensitivity over this period.

The six previously published studies ${ }^{4-9}$ of CPAP in mild OSA show few outcome measures that are consistently sensitive to CPAP therapy after adjustment for a placebo or conservative management. Patel and colleagues ${ }^{3}$ have published a meta-analysis of CPAP trials in OSA and concluded that there was insufficient evidence to show that CPAP reduced sleepiness in patients with mild to moderate levels of OSA.

As this was the first full crossover trial employing a compliance monitored sham CPAP device, it is possible to quantify some important effects. A true physiological doseresponse relationship would be exhibited when highly compliant CPAP users exhibit improvements that are better than all sham CPAP effects. Only the Epworth Sleepiness Scale exhibited this expected pattern. Other significant compliance effects (table 2) do not show this pattern. Patients are not blind to their own compliance levels and are thus able to infer some of the benefit they might expect, independent of the objective benefit CPAP might confer.

This trial shows that CPAP devices set at $<1 \mathrm{~cm} \mathrm{H}_{2} \mathrm{O}$ can be effective placebo controls in a crossover design. Patients tended to prefer sham CPAP and think it better for their sleep. Despite the published concerns ${ }^{11}$ and an observation in a clinical trial ${ }^{31}$ that sham CPAP might have lower compliance rates than active CPAP, no significant difference in compliance rates were observed, nor were patients unblinded..$^{32}$ Unlike other studies using a low pressure CPAP device, ${ }^{28} 29$ this device did not improve the major indices of sleep disordered breathing (table 1). Despite some worsening of overall sleep quality, 19 of 20 daytime outcome measures showed some improvement, addressing worries that sham CPAP might bias in favour of positive findings by worsening daytime function. ${ }^{32}$ Only in the modMWT did sham CPAP tend towards worse daytime function $(p=0.15)$. It is possible that this non-significant effect was due to poorer sleep quality caused by the sham device. These observations lend strength to the conclusion that the placebo effects on the outcome measurements are due to the expectation of benefit of the patients and not a partially beneficial effect on sleep indices of the device.

These findings show that humidified CPAP reduces subjective sleepiness and possibly objective wakefulness in a sample of symptomatic patients with mild OSA. The observed effect on subjective sleepiness could be viewed as a result of statistical chance, but the pattern of improvement is consistent with an hypothesised physiological dose-response relationship. These findings are consistent with the overall picture from other similar trials ${ }^{4-9}$ and a meta-analysis. ${ }^{3}$ The findings of this study, when viewed in context, offer little evidence that CPAP should be routinely used in the treatment of mild OSA, but offer support for the practice of trialling CPAP in patients with mild OSA and severe daytime sleepiness (fig 3 ). ${ }^{33}$

\section{ACKNOWLEDGEMENTS}

The authors thank Gordon Purdie for excellent statistical advice; Margo van den Berg, Karyn O'Keeffe, and Michi Imazu for polysomnograhic expertise; Nick Burgess who was largely responsible for the development of the placebo CPAP interface; and two anonymous reviewers for their excellent and expert advice regarding our manuscript.

\section{Authors' affiliations}

N S Marshall, A M Neill, A J Campbell, D S Sheppard, WellSleep, Department of Medicine, Wellington School of Medicine and Health Sciences, University of Otago, Wellington, New Zealand

N S Marshall, Sleep/Wake Research Centre, Massey University, New Zealand

This research was supported by a grant from the Health Research Council of New Zealand (to AMN) and by a PhD stipend from Massey University (to NSM).

The authors declare that they have no competing interests with regard to this study. The study was not funded by industry interests and the authors at all times retained the exclusive access to the data and rights to publish.

\section{REFERENCES}

1 Young T, Palta M, Dempsey J, et al. The occurrence of sleep disordered breathing among middle aged adults. N Engl J Med 1993;328:1230-5.

2 Sullivan CE, Issa FG, Bethon-Jones M, et al. Reversal of obstructive sleep apnoea by continuous positive airway pressure applied through the nares. Lancet, 1981;i, 862-5.

3 Patel SR, White DP, Malhotra A, et al. Continuous positive airway pressure therapy for treating sleepiness in a diverse population with obstructive sleep apnea: results of a meta-analysis. Arch Intern Med 2003;163:565-71.

4 Barnes M, McEvoy RD, Banks S, et al. Efficacy of positive airway pressure and oral appliance in mild to moderate obstructive sleep apnea. Am J Respir Crit Care Med 2004; 170:656-64.

5 Barnes M, Houston D, Worsnop CJ, et al. A randomized controlled trial continuous positive airway pressure in mild obstructive apnea. Am J Respir Crit Care Med 2002:165:773-80.

6 Engleman HM, Kingshott RN, Wraith PK, et al. Randomised placebo controlled crossover trial of continuous positive airway pressure for mild sleep apnea/hypopnea syndrome. Am J Respir Crit Care Med 1999;159:461-7.

7 Engleman HM, Martin SE, Deary IJ, et al. Effect of CPAP therapy on daytime function in patients with mild sleep apnoea/hypopnoea syndrome. Thorax 1997;52:114-9.

8 Monasterio C, Vidal S, Duran J, et al. Effectiveness of continuous positive airway pressure in mild sleep apnea-hypopnea syndrome. Am J Respir Crit Care Med 2001;164:939-43.

9 Redline S, Adams N, Strauss ME, et al. Improvement of mild sleep disordered breathing with CPAP compared with conservative therapy. Am J Respir Crit Care Med 1998;157:858-65.

10 Karlawish JHT, Pack Al. Addressing the ethical problems of randomized and placebo controlled trials of CPAP. Am J Respir Crit Care Med 2001; 163:809-10.

11 Douglas NJ, Engleman HM, Faccenda JF, et al. The science of designing ethical CPAP trials (letter). Am J Respir Crit Care Med 2002;165:132-3.

12 Massie C, Hart R, Peralez K, et al. Effects of humidification on nasal symptoms and compliance in sleep apnea patients using continuous positive airway pressure. Chest 1999;116:403-8.

13 Neill AM, Wai H, Bannan S, et al. Humidified nasal continuous positive airway pressure in obstructive sleep apnoea. Eur Respir J 2003;22:258-62.

14 Farre R, Hernandez L, Montserrat J, et al. Sham continuous positive airway pressure for placebo-controlled studies in sleep apnoea. Lancet 1999;353:1154.

15 Iber C, Redline S, Kaplan Gilpin A, et al. Polysomnography performed in the unattended home versus the attended laboratory setting - Sleep Heart Health Study methodology. Sleep 2004;27:536-40.

16 Rechtschaffen A, Kales A. A manual of standardized terminology, techniques and scoring system for sleep stages of human subjects. Los Angeles: Brain Information Service/Brain Research Institute, University of California, 1968.

17 American Sleep Disorders Association (ASDA). EEG arousals: scoring rules and examples. Sleep 1992;15:173-84.

18 Johns MW. M, A new method for measuring daytime sleepiness: the Epworth sleepiness scale. Sleep 1991;14:540-5.

19 Doghramii K, Mitler M, Sangal R, et al. A normative study of the maintenance of wakefuness test (MWT). Electroenceph Clin Neurophys 1997:103:554-62.

20 Banks S, Barnes $M$, Tarquinio N, et al. The maintenance of wakefulness test in normal healthy subjects. Sleep 2004;27:799-802.

21 Weaver TE, Laizner AM, Evans LK, et al. An instrument to measure functional status outcomes for disorders of excessive sleepiness. Sleep 1997;20:835-43.

22 Ware J, Sherbourne C. The MOS 36-item short-form health survey (SF-36): conceptual framework and item selection. Med Care 1992;30:473-83.

23 Scott K, Tobias MI, Sarfati D, et al. SF-36 health survey reliability, validity, and norms for New Zealand. Aust NZ J Public Health 1999;23:401-6.

24 Zigmond A, Snaith R. The hospital anxiety and depression scale. Acta Psychiatr Scand 1983;67:367-70.

25 Dinges DF, Powell NB. Microcomputer analyses of performance on a portable, simple visual RT task during sustained operations. Behav Res Methods Instrum Comput 1985;17:652-5.

26 Kazis L, Anderson J, Meenan R. Effect sizes for interpreting changes in health status. Med Care 1989;27(3 Suppl):S178-89.

27 Gander P, Marshall N, Harris R, et al. The Epworth Sleepiness Scale: influence of age, ethnicity, and socio-economic deprivation. Sleep 2005;28:249-53. 
28 Yu B-H, Ancoli-Israel S, Dimsdale JE. Effect of CPAP treatment on mood states in patients with sleep apnea. J Psych Res 1999;33:427-32.

29 Bardwell W, Ancoli-Israel S, Berry C, et al. Neuropsychological effects of one week continuous positive airway pressure treatment in patients with obstructive sleep apnea: a placebo controlled study. Psychsom Med 2001;63:579-84

30 Lamphere J, Roehrs T, Wittig R, et al. Recovery of alertness after CPAP in apnea. Chest 1989;96:1364-7.
31 Jenkinson C, Davies RJO, Mullins R, et al Comparison of therapeutic and subtherapeutic nasal continuous positive airway pressure for obstructive sleep apnoea: a randomised prospective parallel trial. Lancet 1999;353:2100-5.

32 Douglas $\mathrm{N}$. Systematic review of the efficacy of nasal CPAP. Thorax 1998:53:414-5.

33 Engleman H. When does 'mild' obstructive sleep apnea/hypopnea syndrome merit continuous positive airway pressure treatment? Am J Respir Crit Care Med 2002; 165:743-5.

\section{LUNG ALERT}

A novel mechanism in the pathogenesis of $Z \alpha_{1}$-antitrypsin related emphysema

$\Delta$ Mahadeva R, Atkinson C, Li Z, et al. Polymers of Z $\alpha_{1}$-antitrypsin co-localize with neutrophils in emphysematous alveoli and are chemotactic in vivo. Am J Pathol 2005;166:377-86

A lpha-1-antitrypsin (AT) is the predominant proteinase inhibitor in the lung. There are many abnormal variants of AT, and the $\mathrm{Z}$ form is one of the most frequently encountered. Z-AT is prone to polymerisation and the polymeric form aggregates in the liver, causing a plasma deficiency, while monomeric Z-AT is a less effective proteinase inhibitor than normal AT. This quantitative and functional deficiency exposes the lung to damaging proteinases such as neutrophil elastase which results in emphysema. The authors had previously shown that, in addition to localisation in hepatocytes, Z-AT polymers could be found in bronchoalveolar lavage (BAL) fluid and that, in vitro, these polymers attract neutrophils. They postulated that this neutrophil chemotaxis was another mechanism for the emphysema seen in Z-AT homozygotes. The present study was designed to assess the location of polymers in the lung and to test if chemotaxis occurs in vivo.

Tissue was harvested from lungs explanted from Z-AT and normal AT (M-AT) patients with emphysema. ELISA showed that 2.5 -fold more AT was present in M-AT lungs than in Z-AT lungs and that polymers were only found in Z-AT lungs. Immunostaining showed that polymers and neutrophils were both present in the alveolar walls of Z-AT lungs. There was a 4-fold higher number of neutrophils in the Z-AT than the M-AT lungs. In a murine model, BAL fluid samples were taken after installation of polymeric Z-AT and M-AT into the lungs. Polymeric Z-AT produced a significant neutrophil influx into the BAL fluid compared with M-AT.

The findings of Z-AT polymer deposition in alveoli and their chemotactic properties in vivo establish a novel mechanism of emphysema pathogenesis in Z-AT homozygotes and might explain the progression of lung disease in these individuals despite replacement therapy.

J Goldring

Specialist Registrar, Royal Free Hospital, London, UK; ïipgoldring@doctors.org.uk 